\title{
THE PREPARATION AND SOLUBILITY OF LORATADINE-FUMARIC ACID BINARY MIXTURE
}

\author{
FIKRI ALATAS ${ }^{1 *}$, MORISA APRILLIANA ${ }^{1}$, DOLIH GOZALI ${ }^{2}$
}

${ }^{1}$ Department of Pharmaceutical Technology, Faculty of Pharmacy, Universitas Jenderal Achmad Yani, Jl. Terusan Jenderal Sudirman, Cimahi, Indonesia. ${ }^{2}$ Department of Pharmaceutics and Pharmaceutical Technology, Faculty of Pharmacy, Universitas Padjadjaran, Jl. Terusan Jatinangor, Bandung, Indonesia. Email: fikrifaza@yahoo.co.id

Received: 27 September 2016, Revised and Accepted: 11 October 2016

\section{ABSTRACT}

Objective: Loratadine (LOR) is a biopharmaceutics classification system Class II drug that has low solubility and high permeability, so its absorption rate is determined by the dissolution rate. The aim of this study was to prepare an LOR-fumaric acid (LOR-FUM) binary mixture and to investigate its influence on the solubility and dissolution rate of LOR.

Methods: LOR-FUM binary mixture was prepared by the wet grinding method in an equimolar ratio by the addition of two drops of methanol. Characterization of LOR-FUM binary mixture was conducted by the construction of the phase solubility, powder X-ray diffractometry, Fourier transforms infrared spectroscopy, and polarizing microscopy methods. Solubility test was performed in water at room temperature, whereas the in vitro dissolution test was performed in the $\mathrm{pH} 1.2,4.5$, and 6.8 buffer solutions.

Results: The phase solubility curve of LOR in FUM solution is similar to type $\mathrm{B}_{\mathrm{S}}$. The powder X-ray diffraction results show the crystallinity of LOR was changed become more amorphous indicating the salt formation. Fourier transforms infrared spectroscopy (FTIR) spectra of LOR-FUM binary mixture showed the vibration peaks of an imine group on the pyridine ring of LOR and carbonyl group on the FUM are not detected. On the other hand, the polarizing microscope images showed the crystal habit of LOR-FUM binary mixture after recrystallized from methanol contrast to crystal habit of its pure components. The solubility in water of LOR-FUM binary mixture was higher than pure LOR. Amount of LOR released from LOR-FUM binary mixture in $\mathrm{pH} 4.5$ and 6.8 buffer solutions was faster than from pure LOR.

Conclusion: A binary mixture of LOR and FUM may improve the solubility and dissolution rate of LOR.

Keywords: Loratadine, Fumaric acid, Binary mixture, Solubility, Dissolution rate.

(c) 2017 The Authors. Published by Innovare Academic Sciences Pvt Ltd. This is an open access article under the CC BY license (http://creativecommons. org/licenses/by/4. 0/) DOI: http://dx.doi.org/10.22159/ajpcr.2017.v10i1.15400

\section{INTRODUCTION}

Loratadine (LOR) is a second generation antihistamine used to treat allergies without causing drowsiness. The activity mechanism of LOR is a competitive-reversible inhibitor of the histamine receptor tissue, and therefore, this prevents histamine binding and receptor activation [1]. LOR is a drug belonging to a Class II in the biopharmaceutics classification system (BCS), which it has low solubility in water and high permeability [2]. Moreover, LOR is a weak base with a pKa of 5.0 and its solubility is affected by $\mathrm{pH}$ media [3]. Accordingly, LOR has the lowest solubility at pH 7.5 and the highest at pH 1.2 [2]. It is known that a drug with either poor solubility in water or low permeability often causes low bioavailability. The efforts to increase the solubility of LOR have previously been reported, among others, solid dispersion $[4,5]$ and the formation of inclusion complex [6-8].

Modification of solid forms of drugs through the formation of salts or cocrystals is a very good technique to increase the solubility of some drugs [9-11]. Co-crystal is a new solid form consists of a single crystalline phase of several components in a certain stoichiometric ratio, where different types of molecules that interact with hydrogen bonds or other noncovalent bonds [12]. The pyridine ring in their chemical structure LOR as shown in Fig. 1a allows the proton transfer with carboxylic acids to form a salt. Furthermore, $\mathrm{N}$ of the pyridine ring may also act as a hydrogen bond acceptor, allowing the formation of co-crystal when it binds to the hydroxyl group of carboxylic acids. Fumaric acid (FUM) is a dicarboxylic acid that is safe to use orally, and it's widely employed as an excipient in salt or co-crystal formation. FUM (Fig. 1b) can improve the solubility of several drugs through the formation of salt $[13,14]$ and co-crystals [15-18]. Provision of mechanical energy such as grinding in a solid binary mixture with or without the addition of solvents can lead to interactions, among others, an eutectic mixture, salt, or co-crystal formation. The purpose of this research was mainly to prepare a binary mixture of LOR-FUM and to investigate its effect on the solubility and dissolution rate of LOR.

\section{MATERIALS AND METHODS}

Materials

LOR was obtained from Dr. Reddy"s, India. FUM was purchased from Sigma-Aldrich, Singapore (>99\%). All reagents and solvents were purchased from Merck, Indonesia, without any further purification.

\section{Phase solubility test of LOR in FUM solution}

Approximately, $50 \mathrm{mg}$ of LOR put into each vial containing numerous concentrations of FUM solution in water (15-50 mM) and shaken on an orbital shaker at room temperature. The samples in the vials were filtered after $24 \mathrm{hrs}$, and then, the clear filtrate was analyzed by ultraviolet (UV) spectrophotometry method at a wavelength of $283 \mathrm{~nm}$. The phase solubility curve was constructed by plotting the solubility of LOR to the various concentrations of FUM.

Preparation of LOR-FUM binary mixture by wet grinding method A binary mixture of LOR-FUM was prepared by milling an equimolar ratio of LOR-FUM physical mixture in the mortar using a pestle with the addition of two drops of methanol for five minutes. The grinding result was dried and ready to be characterized.

Characterization by powder X-ray diffraction method

The amount of $200 \mathrm{mg}$ each sample (LOR-FUM binary mixture, LOR, and FUM) was placed in a glass container, the sample surface was 
flattened, and then the X-ray powder diffraction pattern was observed using a Philips PW-1710 X-ray diffraction system over a range of angles $2 \theta=5-35^{\circ}$, the speed of $2^{\circ} /$ minutes, a voltage of $40 \mathrm{kV}$, and a current of $30 \mathrm{~mA}$. Then, the powder X-ray diffraction pattern of LOR-FUM binary mixture was compared with the diffraction pattern of pure LOR and FUM.

Characterization by fourier transforms infrared (FTIR) spectroscopy method

Each sample was homogeneously mixed with potassium bromide $(\mathrm{KBr})$ at a weight ratio of 1:5 and conducted by Shimadzu Affinity-1 FTIR spectrophotometer. The $\mathrm{KBr}$ powder is used as a blank for background correction in FTIR, and the samples were scanned in the range of $4000-400 / \mathrm{cm}$. The FTIR spectra of LOR-FUM binary mixture was compared with the FTIR spectra of pure LOR and FUM.

The characterization of LOR-FUM crystal habit by polarizing microscope

As much as $2 \mathrm{mg}$ of LOR-FUM physical mixture was placed on the glass slide, added two drops of methanol, covered with a glass lid, and allowed to recrystallized. The crystal habit after recrystallization was observed using an Olympus BX-53 polarizing microscope equipped with an Optilab Advanced Plus camera and compared with crystal habit of its pure components after recrystallized from the same solvent.

\section{Solubility test}

Solubility test was conducted on the binary mixture of the LOR-FUM and pure LOR in water at room temperature. A certain number of samples were inserted into the vial containing $10 \mathrm{~mL}$ of water and shaken on an orbital shaker. After $24 \mathrm{hrs}$, the sample was filtered and then the filtrate of the sample was diluted and analyzed by a UV spectrophotometer at a wavelength of $283 \mathrm{~nm}$.

\section{In vitro dissolution test}

The in vitro dissolution test was carried out on the LOR-FUM binary mixture and pure LOR in $900 \mathrm{~mL}$ of pH 1.2, 4.5, and 6.8 buffer solutions at $37 \pm 0.5^{\circ} \mathrm{C}$ using a Type II apparatus with 50 rotations/minutes (rpm) of speed for 120 minutes. A $10 \mathrm{~mL}$ of sample was taken after $5,10,15,30,60,90$, and 120 minutes and replaced with the same volume of the buffer solution. Each sample was analyzed by the UV spectrophotometer.

\section{RESULTS AND DISCUSSION}

Phase solubility test

The formation of the salt or co-crystal can be predicted by knowing the type of phase solubility curve. According to Higuchi and Connors, phase solubility curve is a curve that describes the solubility of a substance in the solution at various concentrations of other substances consisting $A_{t}$, $A_{p}, A_{N}, B_{S^{\prime}}$ and $B_{L}$ types [19]. The phase solubility curve of LOR in various concentrations of FUM solution is presented in Fig. 2. The solubility of LOR at 15, 20,30,35, 45, and $50 \mathrm{mM}$ concentrations of FUM solutions were $2.3,3.0,14.6,15.0,6.9$, and $1.9 \mu \mathrm{M}$, respectively, and therefore, the type of phase solubility curve is $B_{S}$. In this type, the solubility of LOR increased sharply in concentrations range of $15-30 \mathrm{mM}$ of FUM solution due to the formation of dissolved new solid form (salt or co-crystal). At concentrations of 30-35 mM, the saturation condition began to occur as a result reaching solubility product constant (Ksp) of LOR-FUM salt or cocrystal. Consequently, the solubility of LOR in the concentration range was fixed. Conversely, the increasing of FUM concentration up to $50 \mathrm{mM}$ caused a decline in FUM solubility, therefore, the precipitated of salt or co-crystal were formed. These results express that between LOR and FUM occurred an interaction. There are two possibilities of the interaction, the first is the transfer of a proton from FUM to LOR to create the salt, and the second is the formation of hydrogen bonds between the LOR and FUM to assemble the co-crystal.

\section{Preparation of LOR-FUM binary mixture}

The grinding of a physical mixture of two solid substances either with or without the addition of solvent can lead to the formation of salt [20] or co-crystal [21]. In this study, the grinding was conducted on the

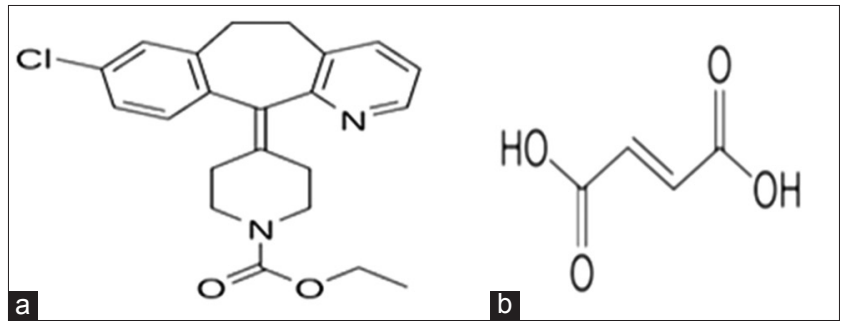

Fig. 1: Chemical structures of (a) loratadine and (b) fumaric acid

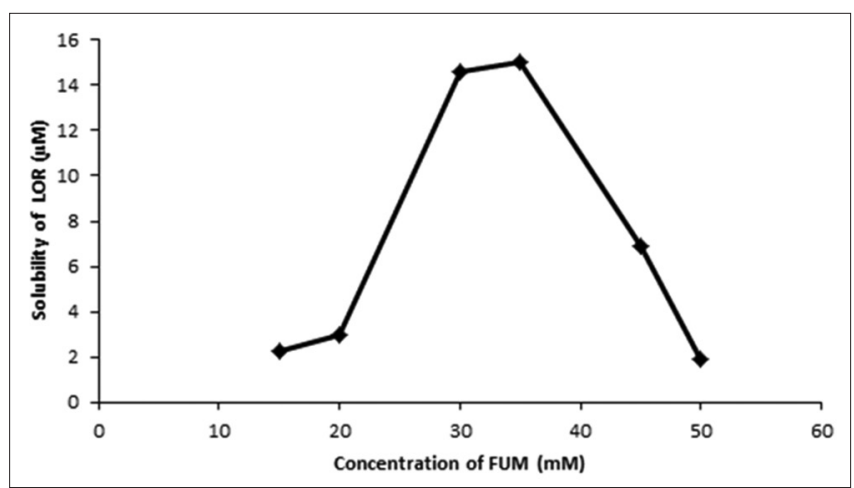

Fig. 2: Phase solubility curve of loratadine in various concentrations of fumaric acid solution in water

equimolar of LOR-FUM physical mixture with the addition of a few drops of methanol, and this grinding technique is known as wet grinding or solvent-drop grinding method [22]. The purpose of the addition of methanol was to accelerate the two components interaction, as a result of the rapid achievement of the amorphous state which causes each component to be more reactive [23]. Besides increasing the interaction, methanol was also able to dissolve of LOR and FUM. At the beginning of the wet grinding, a sticky mixture was created that indicates the amorphous state formation, followed by the recrystallization process into a dry solid form.

Characterization by powder X-ray diffraction method

The powder X-ray diffractometry is a very powerful method to analyze the formation of a new crystalline phase [24]. The powder X-ray diffraction pattern of LOR, FUM, and LOR-FUM binary mixture are shown in Fig. 3. The powder X-ray diffraction pattern of LOR raw material shows main peaks at $6.4,12.7,15.1,19.6,21.2,23.8$, and $30.5^{\circ}$ of $2 \theta$ angle, and this diffraction pattern is consistent with the crystal structure of LOR with BEQGIN reference code [25]. The FUM raw material has main peaks of powder X-ray diffraction at 18.6, $22.8,24.5,25.9,28.9$, and $29.5^{\circ} 2 \theta$ angle, and the diffraction pattern conform to form $\alpha$ of FUM with FUMAAC reference code [26]. The initial characterization of the formation of new solid form was conducted by comparing the powder X-ray diffraction pattern of a binary mixture of LOR-FUM with its parent components. The difference between powder X-ray diffraction pattern of binary mixture and powder X-ray diffraction pattern of each component indicates the formation of cocrystal [27]. The powder X-ray diffraction pattern of a binary mixture between LOR and FUM in Fig. 2 was not different from the powder X-ray diffraction pattern of pure LOR and FUM. The main peaks of LOR and FUM still appear in the diffraction pattern of LOR-FUM binary mixture. Nevertheless, the powder X-ray diffraction pattern of LOR-FUM binary mixture showed the changes become more amorphous, which is denoted by lowering the intensity of the main peaks. Decreasing the intensity peaks is one of the characteristics of salt formation, and therefore, it is suspected to occur due to the formation of LOR-FUM salt.

\section{Characterization by FTIR method}

IR can also be employed to investigate the interaction between two or more components. Accordingly, it is capable of characterizing the 
formation of co-crystals, salts, polymorphs, hydrates, and solvates [28]. The co-crystal exists due to the existence of the hydrogen bonds, whereas the salt occurs due to the occurrence of proton transfer and acceptance between two interacted substances. In Fig. 4., LOR has a characteristic absorption band at $1705 \mathrm{~cm}^{-1}$ due to the stretching vibration of carbonyl $(\mathrm{C}=0)$ group in the ester and $1643 \mathrm{~cm}^{-1}$ due to stretching vibration of imine group $(\mathrm{C}=\mathrm{N})$ in the pyridine ring. FUM has a wide band in the region $3500-3000 \mathrm{~cm}^{-1}$ caused by stretching vibration $-\mathrm{OH}$ group and $1678 \mathrm{~cm}^{-1}$ due to the stretching vibration of the carbonyl group. FTIR spectra of LOR-FUM binary mixture showed the vibration peaks of an imine group in the pyridine ring of LOR and carbonyl group in the FUM are not detected. Accordingly, the undetected of peaks in these groups may be due to the occurrence of proton transfer from the carboxylic group of FUM to the imine group of the pyridine ring of LOR to form a salt.

The characterization of LOR-FUM crystal habit by polarizing microscope

The polarizing microscopy method is able to indicate the formation a new solid form through changes in the crystal habit [29]. The polarizing microscope is capable of exhibiting the difference in the crystal habit between LOR-FUM binary mixture and its pure components after recrystallized from methanol. Fig. 5 shows that the LOR has anhedralshaped crystal habit, whereas FUM has dendritic-shaped crystal habit.

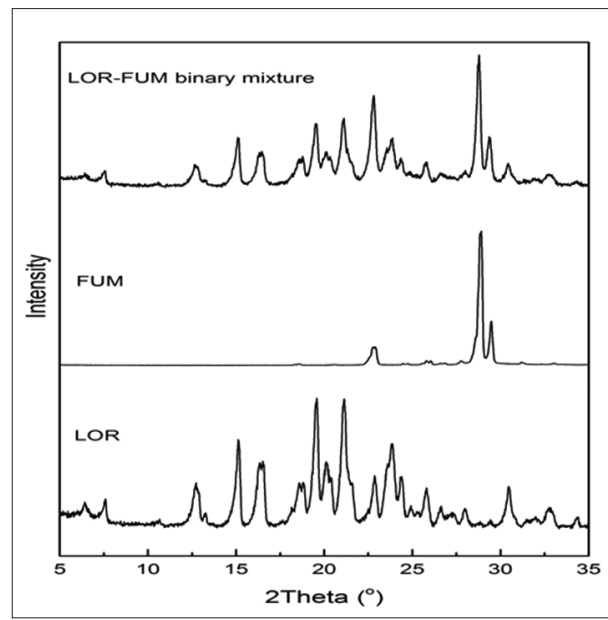

Fig. 3: Powder X-ray diffraction pattern of loratadine (LOR), fumaric acid (FUM), and LOR-FUM binary mixture

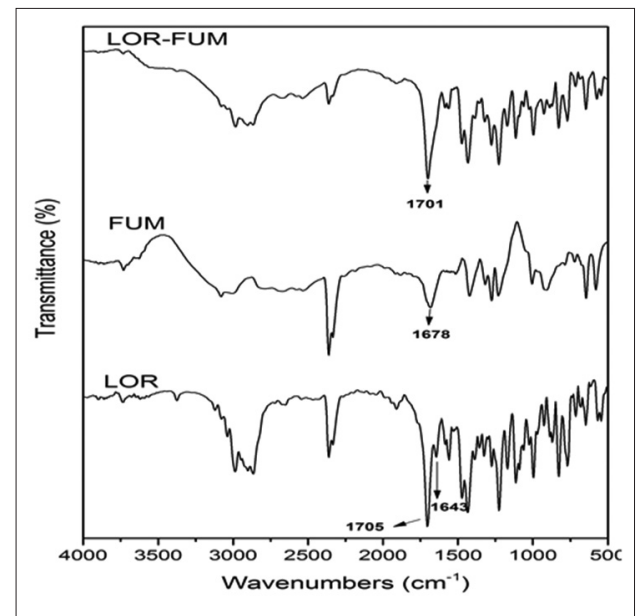

Fig. 4: Fourier transforms infrared spectroscopy spectra of loratadine (LOR), fumaric acid (FUM), and LOR-FUM binary mixture
However, the recrystallization of LOR-FUM binary mixture produced different habits from its pure components. This difference may be due to the formation of LOR-FUM salt.

\section{Solubility test}

The assay of LOR in a mixed solution of LOR and FUM from solubility and dissolution test results was carried out by direct UV spectrophotometry method. FUM has no absorption at the maximum absorption wavelength of LOR $(\lambda=283 \mathrm{~nm})$, so it does not interfere with the absorption of LOR. The influence of a binary mixture of LOR-FUM on the solubility of LOR can be determined by comparing the solubility of pure LOR and LOR-FUM binary mixture in water (neutral medium). The solubility of LOR from the LOR-FUM binary mixture in water at room temperature was 84 -folds higher than pure LOR. The significant improvement of the LOR solubility from this binary mixture caused by increasing the amount of protonated $\mathrm{N}$ of the pyridine ring because of the existence of FUM.

\section{In vitro dissolution test}

The gastrointestinal absorption rate of BCS Class II drugs is limited by its dissolution rate, and consequently, the drugs in this class will be quickly absorbed when they have a rapid dissolution rate [5]. In vitro dissolution test was performed on pure LOR and LOR-FUM binary mixture in $\mathrm{pH} 1.2,4.5$, and 6.8 buffer solutions at $37^{\circ} \mathrm{C}$ to approach the real condition in the gastrointestinal tract [30]. The dissolution profiles

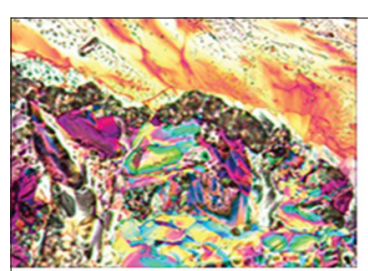

LOR

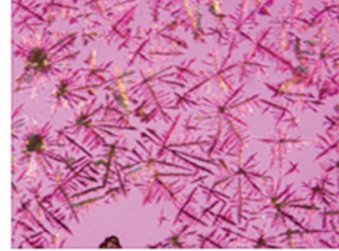

FUM

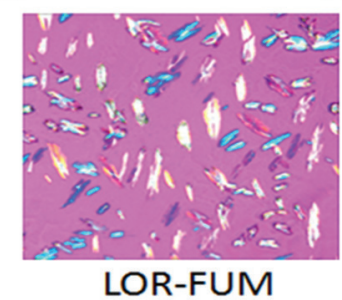

Fig. 5: Polarizing microscope photo of loratadine (LOR), fumaric acid (FUM), and LOR-FUM after recrystallized from methanol solvent

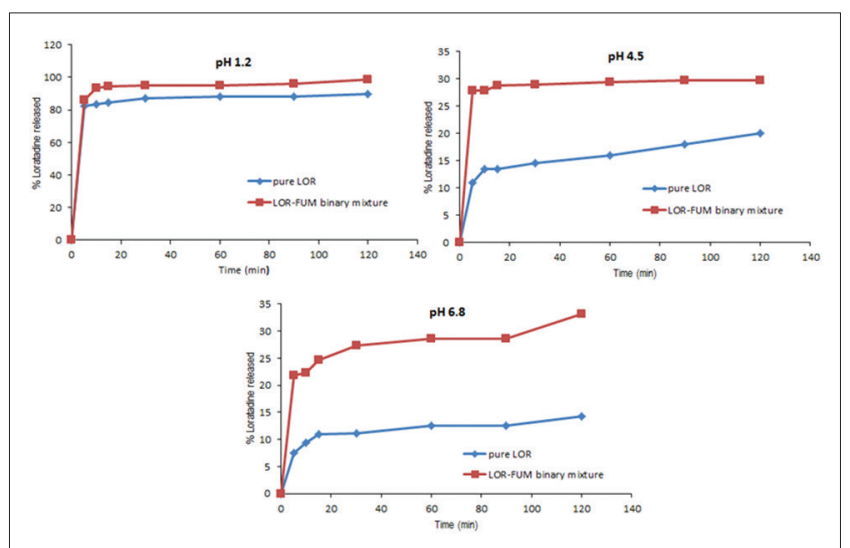

Fig. 6: The dissolution profile of pure loratadine (LOR) and LOR-fumaric acid binary mixture in pH 1.2, 4.5, and 6.8 buffer solutions 
of LOR from the LOR-FUM binary mixture and pure LOR in the all $\mathrm{pH}$ buffer solutions are shown in Fig. 6. Because of the solubility of LOR decrease by increasing of $\mathrm{pH}$ [3], the amount of LOR released both pure LOR and LOR-FUM binary mixture in $\mathrm{pH} 1.2$ is faster than the others. The percentage of LOR dissolved from both samples after 5 minutes have reached more than $80 \%$ in $\mathrm{pH} 1.2$; therefore, the difference of dissolution rate in between LOR-FUM binary mixture and pure LOR was not significant. On the contrary, the percentage of drug dissolved after 5 minutes from LOR-FUM binary mixture in the $\mathrm{pH} 4.5$ and 6.8 buffer solutions was higher than from pure LOR. In these $\mathrm{pH}$, the amount of LOR ionized from LOR-FUM binary mixture was higher than pure LOR, and this may be related to the LOR-FUM salt formation.

\section{CONCLUSION}

A binary mixture of LOR-FUM had been prepared by the wet grinding method and characterized by construction of the phase solubility, powder X-ray diffraction, FTIR spectroscopy, and microscopy methods. The phase solubility curve of LOR in numerous concentrations of FUM solution is $B_{S}$ type, and consequenly, it is supposedly assembled a salt or a co-crystal between LOR and FUM. Although there is no difference in the powder X-ray diffraction pattern between the LOR-FUM binary mixture and its pure constituents, the intensity of this binary mixture is lower than its pure components. The characterization results show the differences in the FTIR spectra and crystal habit between the LOR-FUM binary mixture and their pure components. The LOR-FUM binary mixture may improve the solubility of LOR in water and the dissolution rate in $\mathrm{pH} 4.5$ and 6.8 buffer solutions. The enhancement of the solubility and the dissolution rate of LOR may be due to the salt formation.

\section{ACKNOWLEDGMENT}

We thank the Lembaga Penelitian dan Pengabdian kepada Masyarakat, Universitas Jenderal Achmad Yani, for funding the research.

\section{REFERENCES}

1. Simons FE. Comparative pharmacology of $\mathrm{H} 1$ antihistamines: Clinical relevance. Am J Med 2002;113 Suppl 9A:38S-46.

2. Khan MZ, Rausl D, Zanoski R, Zidar S, Mikulcic JH, Krizmanic L, et al. Classification of loratadine based on the biopharmaceutics drug classification concept and possible in vitro-in vivo correlation. Biol Pharm Bull 2004;27(10):1630-5.

3. Popovic G, Cakar M, Agbaba D. Acid-base equilibria and solubility of loratadine and desloratadine in water and micellar media. J Pharm Biomed Anal 2009;49(1):42-7.

4. Sathyaraj A, Palraja M. Preparation and comparative evaluation of loratadine solid dispersions with various binders by spray drying technique. Int J Res Pharm Chem 2012;2(1):37-45.

5. Frizon F, Eloy JO, Donaduzzi CM, Mitsui ML, Marchetti JM. Dissolution rate enhancement of loratadine in polyvinylpyrrolidone K-30 solid dispersions by solvent methods. Powder Technol 2013;235:532-9.

6. Lin S, Lin H, Lin C. Thermodynamic study of grinding-induced loratadine inclusion complex formation using thermal analysis and curve-fitted FTIR determination. In: Moreno JC, editor. Thermodynamics - Physical Chemistry of Aqueous Systems. Shanghai: InTech; 2011. p. 317-26.

7. Pooja S, Meenakshi B, Shruti S. Physicochemical characterization and dissolution enhancement of loratadine - Hydroxypropyl- $\beta$-cyclodextrin binary systems. J Pharm Sci Res 2011;3(4):1170-5.

8. Szabados-Nacsa A, Sipos P, Martinek T, Mándity I, Blazsó G, Balogh Á, et al. Physico-chemical characterization and in vitro/in vivo evaluation of loratadine: Dimethyl-ß-cyclodextrin inclusion complexes. J Pharm
Biomed Anal 2011;55(2):294-300.

9. Elder DP, Holm R, Diego HL. Use of pharmaceutical salts and cocrystals to address the issue of poor solubility. Int J Pharm 2013;453(1):88-100.

10. Shevchenko A, Bimbo LM, Miroshnyk I, Haarala J, Jelínková K, Syrjänen K, et al. A new cocrystal and salts of itraconazole: Comparison of solid-state properties, stability and dissolution behavior. Int J Pharm 2012;436(1-2):403-9.

11. Raghuram M, Alam MS, Prasad M, Khanduri CH. Pharmaceutical cocrystal of prulifloxacin with nicotinamide. Int J Pharm Pharm Sci 2014;6(10):180-4.

12. Rodríguez-Hornedo N, Nehm SJ, Jayasankar A. Cocrystals: Design, properties and formation mechanisms. Encyclopedia of Pharmaceutical Technology. London: Taylor \& Francis; 2007. p. 615-35.

13. Sanphui P, Tothadi S, Ganguly S, Desiraju GR. Salt and cocrystals of sildenafil with dicarboxylic acids: Solubility and pharmacokinetic advantage of the glutarate salt. Mol Pharm 2013;10(12):4687-97.

14. Tsutsumi S, Iida M, Tada N, Kojima T, Ikeda Y, Moriwaki T, et al. Characterization and evaluation of miconazole salts and cocrystals for improved physicochemical properties. Int J Pharm 2011;421(2):230-6.

15. Martin FA, Pop MM, Borodi G, Filip X, Kacso I. Ketoconazole salt and co-crystals with enhanced aqueous solubility. Cryst Growth Des 2013;13(10):4295-304

16. Shevchenko A, Miroshnyk I, Pietilä LO, Haarala J, Salmia J, Sinervo K, et al. Diversity in itraconazole cocrystals with aliphatic dicarboxylic acids of varying chain length. Cryst Growth Des 2013;13(11):4877-84.

17. Smith AJ, Kavuru P, Wojtas L, Zaworotko MJ, Shytle RD. Cocrystals of quercetin with improved solubility and oral bioavailability. Mol Pharm 2011;8(5):1867-76.

18. Hiendrawan S, Hartanti AW, Veriansyah B, Widjojokusumo E, Tjandrawinata RR. Solubility enhancement of ketoconazole via salt and cocrystal formation. Int J Pharm Pharm Sci 2015;7(7):160-4.

19. Higuchi T, Connors KA. Phase solubility techniques. In: Advances in Analytical Chemistry and Instrumentation. New York: Jonh Wiley \& Sons; 1965. p. 117-212.

20. Trask AV, Haynes DA, Motherwell WD, Jones W. Screening for crystalline salts via mechanochemistry. Chem Commun (Camb) 2006;1:51-3.

21. Karki S, Friscic T, Jones W, Motherwell WD. Screening for pharmaceutical cocrystal hydrates via neat and liquid-assisted grinding. Mol Pharm 2007;4(3):347-54.

22. Trask AV, Motherwell WD, Jones W. Solvent-drop grinding: Green polymorph control of cocrystallisation. Chem Commun (Camb) 2004; 7:890-1.

23. Shan N, Toda F, Jones W. Mechanochemistry and co-crystal formation: Effect of solvent on reaction kinetics. Chem Commun (Camb) 2002;20:2372-3.

24. Zaini E, Sumirtapura YC, Soewandhi SN, Halim A, Uekusa H, Fujii K. Cocrystalline phase transformation of binary mixture of trimethoprim and sulfamethoxazole by slurry technique. Asian J Pharm Clin Res 2010;3(4):26-9.

25. Kaminski JJ, Carruthers NI, Wong SC, Chan TM, Billah MM, Tozzi S, et al. Conformational considerations in the design of dual antagonists of platelet-activating factor (PAF) and histamine. Bioorg Med Chem 1999;7(7):1413-23.

26. Brown CJ. The crystal structure of fumaric acid. Acta Cryst 1966;21:1-5.

27. Sanphui P, Goud NR, Khandavilli UB, Nangia A. Fast dissolving curcumin cocrystals. Cryst Growth Des 2011;11(9):4135-45.

28. Van Eerdenbrugh B, Taylor LS. Application of mid-IR spectroscopy for the characterization of pharmaceutical systems. Int $\mathrm{J}$ Pharm 2011;417(1-2):3-16

29. Setyawan D, Sari R, Yusuf H, Primaharinastiti R. Preparation and characterization of artesunate-nicotinamide cocrystal by solvent evaporation and slurry method. Asian J Pharm Clin Res 2014;7 Suppl 1:62-5

30. Tubic-Grozdanis M, Bolger MB, Langguth P. Application of gastrointestinal simulation for extensions for biowaivers of highly permeable compounds. AAPS J 2008;10(1):213-26. 